\title{
Inhibition of PI3K/AKT molecular pathway mediated by membrane estrogen receptor GPER accounts for cryptotanshinone induced antiproliferative effect on breast cancer SKBR-3 cells
}

Danning Shi ( $\sim$ shiney@bucm.edu.cn )

Beijing University of Chinese Medicine

\section{PIWEN ZHAO}

Beijing University of Chinese Medicine

LIXIA CUI

Beijing University of Chinese Medicine

LIPING SUN

Beijing University of Chinese Medicine

HONGBO LI

Beijing University of Chinese Medicine

JIANZHAO NIU

Beijing University of Chinese Medicine

MENG CHEN

Beijing University of Chinese Medicine

Research article

Keywords: cryptotanshinone, GPER, breast cancer, cell cycle, cyclin, PI3K/AKT, molecular pathway

Posted Date: September 17th, 2019

DOI: https://doi.org/10.21203/rs.2.14470/v1

License: (c) (1) This work is licensed under a Creative Commons Attribution 4.0 International License.

Read Full License

Version of Record: A version of this preprint was published at BMC Pharmacology and Toxicology on May 1st, 2020. See the published version at https://doi.org/10.1186/s40360-020-00410-9. 


\section{Abstract}

Background: Breast cancer is the most frequently diagnosed cancer among women and the second leading cause of cancer death. Especially nuclear estrogen receptor (nER) negative breast cancer is always with much poor prognosis. In recent years, it was reported that membrane $\mathrm{G}$ protein coupled estrogen receptor (GPER), a newly recognized estrogen receptor took essential part in the development and treatment of breast cancer, especially nER negative breast cancer. The present study aimed to investigate the anti nER negative breast cancer effect and its possible molecular pathway of cryptotanshinone (CPT), an important active compound of traditional Chinese medicine Danshen.

Methods: The following in vitro tests were performed in nER negative but GPER positive breast cancer SKBR-3 cells. The effect of CPT on cell proliferation rate and cell cycle distribution was evaluated by MTT cell viability test and flow cytometry assay. The role of PI3K/AKT pathway and the mediated function of GPER were tested by western blot and immunofluorescence. Technique of gene silence and the specific GPER agonist G-1 and antagonist G-15 were employed in the experiments to further verify the function of GPER in mediating the anticancer role of CPT.

Results: The results showed that proliferation of SKBR-3 cells could be blocked by CPT in a time and dose dependent manner. CPT also exerted antiproliferative activities by arresting cell cycle progression in G1 phase and down regulating the expression level of cyclin A, cyclin B, cyclin D and cyclin-dependent kinase 2 (CDK2). The antiproliferative effect of CPT was further enhanced by G1 and attenuated by G15. Results of western blot and immunofluorescence both showed that PI3K and p-AKT protein expression could be down regulated by CPT and such effects were mediated by GPER which is further demonstrated by GPER gene silence test.

Conclusion: Our present study shows that the antiproliferative action of CPT on SKBR-3 cells is realized by inhibition of GPER mediated PI3K/AKT pathway. These findings deserve further validation for serving as useful therapeutic targets.

\section{Background}

Breast cancer becomes one of the most common malignancies and an important public health problem worldwide in recent years. It also stands in the second place among the most common causes of death from cancer in women nowadays [1]. The following approaches including surgery, radiation-therapy and chemo-therapy have contributed much to increase the survival rate of patients with breast cancer[2]. But nevertheless, the prognosis of some kinds of breast cancer, especially nuclear estrogen receptor negative breast cancer remains poor[3]. So effective therapeutic methods against breast cancer are still required and need to be further explored.

Besides the above clinical approaches in breast cancer treating, traditional Chinese medicine as well as its active components could also play prominent roles in breast cancer treatment so far. As major bioactive compounds in Salvia miltiorrhiza Bunge (Danshen), Tanshinones have been proved to exert inhibitory action on proliferation and migration of breast cancer cells effectively[4-7]. Recently, cryptotanshione (CPT), another 
important kind of active component in Danshen began to attract much attention due to its anti-inflammatory [8], anti-bacterium[9] and antitumor effects[10-12]. Among which the antitumor function has been paid much concern and it has also been documented that CPT could inhibit proliferation and promotes apoptosis of breast cancer cells[12]. However, the pharmacological mechanism, especially the molecular pathway of its effect still remains unclear and requires further study.

Estradiol is a key hormone in breast cancer development[13]. Estrogen receptor (ER) plays vital roles in mediating the action of estrogen on proliferation of cells in different target tissues under both physiological and pathological conditions [14]. A key function of estrogen receptor has been reported in the proliferation and migration of breast cancer cells [15]. Besides the classical nuclear estrogen receptor $\alpha$ and $\beta$, recently a new membrane estrogen receptor known as G protein-coupled estrogen receptors (GPER) attracted much attention and has now been recognized as a major mediator of estrogen's rapid cellular effects throughout the body[16, 17]. For only approximately $70 \%$ of primary breast cancers are nER positive, GPER is now considered to be a possible target point in cancer therapy, especially in nER negative breast cancer cells. GPER and its mediated signal pathway has been confirmed to be involved in the proliferation and migration of breast cancer cells[18, 19].

Crucially, the proliferation of cancer cells depends on the cell cycle. Cell cycle regulation is the major regulatory mechanism of cell growth which is modulated by several types of cyclin and cyclin-dependent kinase (CDK). Cell cycle arrest has been found in a number of cell lines after chemotherapy and its dysregulation is a trait of tumor cells[20]. Antitumor agents may cause a cell cycle arrest in various phases by regulating the cell cycle machinery[21]. As regards to the molecular mechanism of proliferation and cell cycle regulation of human cells, the phosphatidylinositide 3-kinase(PI3K)/AKT signaling pathway has been reported to be an essential route and in some estrogen related cancers it is mediated by membrane GPER [22]. Akt is a downstream mediator which could be activated by PI3K signaling and initiates a series of biological effects on proliferation and apoptosis in breast cancer cells. Since activation of PI3K/AKT signal transduction is a main force that drives cell growth, down-regulation or blockage of PI3K and AKT function might be crucial for cancer therapy[23].

Above all, in the present study, the GPER mediated antiproliferation effect via PI3K/AKT molecular pathway induced by cryptotanshinone will be studied in nER negative breast cancer SKBR-3 cells. GPER as a valid option for potential therapeutic target in nuclear ER negative breast cancer will be expected.

\section{Results}

CPT suppressed SKBR-3 cell growth dose and time dependently.

The Chemical structure of CPT is shown in Fig 1A. The viability of SKBR-3 cells obviously decreased at $24 \mathrm{~h}$ after CPT treatment (Fig 1B). By using MTT cell viability assay, we further demonstrated that CPT could significantly decrease the viability of SKBR-3 cells in a dose and time dependent manner (Fig 1C). Treatment with $1 \mu \mathrm{M}, 2.5$ $\mu \mathrm{M}, 5 \mu \mathrm{M}$ and $10 \mu \mathrm{M}$ CPT for $24 \mathrm{~h}$ and $48 \mathrm{~h}$ resulted in $87.8 \%$ and $73.7 \%, 67.2 \%$ and $54.0 \%, 61.4 \%$ and $48.3 \%$ 口 $\mathbf{4 8 . 5 \%}$ and $34.8 \%$ cell viability compared with control group respectively. 
Figure 1. Inhibitory effect of CPT on SKBR-3 cell viability. (A) Chemical structure of Cryptotanshinone (CPT, molecular weight $=296.35 \mathrm{~g} / \mathrm{mol}$ ). (B) SKBR-3 cell morphology under inverted microscope (magnification, $\times 200$ ) after CPT treating for $24 \mathrm{~h}$ with $0.2 \%$ DMSO as control. Scale bar represents $10 \mu \mathrm{m}$. (C) SKBR-3 cells were treated with indicated concentrations of CPT for $24 \mathrm{~h}$ or $48 \mathrm{~h}$ and cell viability was tested using MTT viability assay. The results are means of three independent replicates \pm S.D. ${ }^{* *} P$ values $\square 0.01$ vs control group were considered as statistically significant.

CPT inhibits SKBR-3 cells viability through a GPER-mediated manner.

To further demonstrate the requirement of a functional GPER in CPT induced inhibitory effect on cells viability, we knocked down GPER expression using GPER siRNA transfection. The result showed a low expression of GPER in SKBR-3 cells after transfected with siRNA targeting GPER (Fig 2A). Then we tested cell viability affected by CPT using the GPER knocked down cells. The results indicated that knockdown of GPER abolishes the decrease of cell viability induced by CPT treatment for 48h (Fig 2B). In addition, after using the GPER selective agonist G-1 or antagonist G-15 for 48h, the result showed that viability of SKBR-3 cells was significantly lower when treated by $5 \mu \mathrm{M}$ CPT together with G1 while much higher when treated together with G15 (Fig 2C).

Figure 2. CPT inhibits SKBR-3 cell viability via GPER mediated pathway. (A) GPER protein expression level in GPER siRNA transfected SKBR-3 cells were tested by Western blot with non-siRNA transfection as control. ** $P$ 0.01 vs control group. (B) Viability of GPER knocked down SKBR-3 cells was tested by MTT viability assay. The results are means of three independent replicates \pm S.D. $* * P] 0.01$ vs control group or $\Delta \Delta_{P} 0.01$ vs non-siRNA group were considered as statistically significant. (C) Viability of SKBR-3 cells treated by CPT with G1 or G15 was also tested by MTT viability assay. The results are means of three independent replicates \pm S.D. ${ }^{* *} P[0.01$ vs control group or $\Delta_{P} 0.01$ vs $5 \mu \mathrm{M}$ CPT treated group were considered as statistically significant. 
CPT induces cell cycle arrest in SKBR-3 cells.

Cell cycle is the basis of cell proliferation. Inducing cell cycle arrest could be a crucial way to inhibit the development of tumor cells. As shown in Fig 3, the effect of CPT on cell cycle progression was analyzed using FCM following $48 \mathrm{~h}$ CPT treating. An increase of the percentage of SKBR-3 cells in G1-phase to $71.86 \pm 3.03 \%$ and $77.35 \pm 5.56 \%$ was observed using $5 \mu \mathrm{M}$ and $10 \mu \mathrm{M}$ CPT respectively, compared with the control group, $65.83 \pm 3.47 \%$. In the meanwhile, percentage of SKBR-3 cells in G2-phase was decreased from $15.86 \pm 1.02 \%$ in control group to $7.87 \pm 0.75 \%$ and $6.88 \pm 0.87 \%$ using $5 \mu \mathrm{M}$ and $10 \mu \mathrm{M}$ CPT respectively. The results revealed that CPT induced suppression of breast cancer SK-BR3 cell growth might be associated with a GPER mediated G1phase arrest.

Figure 3. Effect of CPT treating for $48 \mathrm{~h}$ on cell cycle distribution in SKBR-3 cells. The results are means of three independent replicates \pm S.D. $* P] 0.05$ or ${ }^{* *} P$ values $[0.01$ vs control group were considered as statistically significant.

CPT regulates the expression levels of cell cycle-associated proteins in SKBR-3 cells.

Following treatment with CPT on SKBR-3 cells for $48 \mathrm{~h}$, the expression levels of cyclin A, cyclin B, cyclin D and CDK2 decreased in a dose-dependent manner (Fig 4).

Figure 4. The expression level of cell cycle-associated proteins in SKBR-3 following treatment with CPT for $48 \mathrm{~h}$. The results are means of three independent replicates \pm S.D. $* P \| 0.05$ or $* * P$ values $\square 0.01$ vs control group were considered as statistically significant.

Inhibition of PI3K/AKT signaling pathway by CPT in SKBR-3 cells.

In order to make it clear that whether GPER mediated PI3K/AKT signaling pathway is involved in CPT induced cell viability inhibition and cell cycle arrest in SKBR- 3 cells, we tested the expression of PI3K and p-AKT after CPT treating. Image analysis demonstrated a clear dose-dependent reduction in p-AKT and PI3K expression in response to CPT treating (Fig.5)

Figure 5. The expression level of PI3K and p-AKT in SKBR-3 cells treated by CPT for $48 \mathrm{~h}$. The results are means of three independent replicates \pm S.D. $* P 00.05$ vs control group were considered as statistically significant.

Regulation of PI3K, p-AKT and cell cycle-associated proteins expression by CPT in SKBR-3 cells is realized in a GPER mediated manner. 
Treatment of SKBR-3 cells with G-1 or G-15 together with 5 $\mu$ M CPT resulted in a decreasing and increasing expression level of PI3K and p-AKT respectively as shown in Fig 6A. To further confirm the effects of CPT on downstream proteins of PI3K, a specific PI3K inhibitor-LY294002 was used. Treating with LY294002 resulted in a reduction of p-AKT and cell cycle associated proteins (Fig.6B). These findings support the hypothesis that inhibition of SKBR-3 by CPT is realized by the GPER mediated suppression of PI3K/AKT signaling.

Expression of PI3K was also shown using IF assay in Fig 6C. After analyzing by image J software, the mean fluorescence intensity was shown in Fig 6D. The result confirmed that expression of PI3K could be inhibited by CPT in a dose dependent manner. When G-1 was used together with CPT, the expression of PI3K further decreased. On the contrary, PI3K expression increased after using G-15.

Figure 6. (A) The expression level of cyclin A, cyclin B, cyclin D, CDK2, PI3K and p-AKT following G-1, G-15 treating together with $5 \mu \mathrm{M}$ CPT. (B) The expression level of cyclin A, cyclin B, cyclin D, CDK2 and p-AKT following LY294002 treating together with 5 $\mu \mathrm{M}$ CPT. (C) Effect of CPT treatment for $24 \mathrm{~h}$ on PI3K expression by using immunofluorescence assay. Nuclei were counterstained with DAPI (blue). (magnification, $\times 200$, Scale bar represents $10 \mu \mathrm{m})$. (D) The mean fluorescence intensity of PI3K by IF assay was analyzed using Image J software. The results are means of three independent replicates \pm S.D. **P 0.01 or *P 0.05 vs control group, $\triangle \triangle \mathrm{P} 0.01$ vs $5 \mu \mathrm{M} C \mathrm{CPT}$ treating group were considered as statistically significant.

\section{Discussion}

Breast cancer is the most common cancer that displays a high mortality rate among women. And there is an estimated 5-year survival rate of $80 \%$ in developed countries to below $40 \%$ for developing countries[25]. Clinical and laboratory evidences have indicated that nuclear ER negative breast cancer was resistant to several anticancer drugs[26, 27]. In view of its poor prognosis, clinical therapy is a big challenge. It is eager to find much effective treating methods towards $\mathrm{nER}$ negative breast cancer.

Notably, it was reported that many kinds of breast cancer cells, including $\mathrm{nER}$ negative breast cancer cells express membrane GPER[28]. Researches on the effect of GPER in breast cancer treating claimed that GPER might function as a tumor suppressor in breast cancer cells in vitro[19, 29-32]. GPER is a seven-transmembrane protein which is shown to mediate rapid non-genomic signal transduction responding to estrogen[17]. The discovery of GPER as a receptor for estrogens brings a new breakthrough point to explain the mechanism of the effects of estrogen or estrogenic substances on growth and migratory process, especially in the absence of classic nER. Regarding the anticancer agent, in recent years, traditional Chinese medicine has attracted much 
attention worldwide. There has been an increasing interest in the anticancer activity of tanshinones including cryptotanshione, a cell permeable diterpene quinone[12] which are the major bioactive compounds of Danshen. Our current research focused on the anti breast cancer effects of cryptotanshione via the GPER mediated molecular pathway in nER negative SKBR-3 cells.

Our study indicated that CPT are capable of activating GPER leading to SKBR-3 cell proliferation inhibition and cell cycle arrest in a time and concentration dependent manner. Concretely, the cell viability assay indicated that CPT could inhibit proliferation of SKBR-3 cells. Silencing GPER with specific siRNA abolished the decrease in cell viability induced by CPT which indicated that activation of GPER is an important mechanism that may contribute to the anticancer activity of CPT in SKBR-3 cells. Application of GPER selective agonist G-1 and antagonist G15 gave us more information about the function of GPER mediating the effect of CPT on cell proliferation. G-1 was reported to suppress proliferation of ovarian and breast cancer cells in vitro [33]. G-15 was reported to inhibit GPER-mediated function on cell proliferation in vitro [34] In accordance with these previous studies, the inhibitory effects on cell proliferation induced by CPT was further increased with the application of G1 and decreased with G15.

A large section of research has indicated that cell cycle arrest was linked to cell proliferation in cancer cells closely[23, 35, 36]. It is of much significance to deregulate the cell cycle in treatment of cancer. Therefore, elucidating the inhibitory activity on cell cycle by CPT could help us to explain the mechanism of its anticancer effect. The results from our study claimed that the protein expression levels of cyclin A, cyclin B, cyclin D and CDK2 were dose-dependently decreased by treatment of CPT. It is a recognized concept that cells initiate fate decisions in G1-phase. In early G1-phase, cyclin D triggers the cell cycle to the S phase and the DNA replication could be induced [37]. So CPT induced cyclin D reduction in SKBR-3 cells might cause a G1-phase arrest which was further demonstrated by FCM test. The expression of cyclin A was related to S phase and cyclin B to G2/M phase. Our result indicated that the expression of both cyclin A and cyclin B were inhibited by CPT in a dosedependent manner. It might also be caused by the G1-phase arrest in cell cycle since large amount of cells stopped at G1-phase and the cell cycle could not proceed as the original.

As a key regulator of cell proliferation and survival, the PI3K/AKT pathway is critical for cell cycle progression. AKT, the Ser/Thr kinase, was the core component of this cascade of events and activation of PI3K results in the phosphorylation of AKT. That is to say, PI3K-produced phospholipids favor the membrane recruitment of AKT which is itself further activated by either the 3-phospho-inositidedependent protein kinase 1 (PDK1) or by the mechanistic target of rapamycin (mTOR) complex 2 (mTORC2) [7, 38]. Previously, it is reported that p-AKT signaling is much more activated in breast cancer cells and targeting PI3K/AKT signaling may be considered a prime strategy in cancer treatment[39]. Consistent with these studies, $48 \mathrm{~h}$ treatment with CPT showed an inhibitory effect on the expression of both PI3K and p-AKT. PI3K/AKT favors cell proliferation through direct regulation of cell cycle proteins, the cyclins. [23, 40]. Our current research, also indicated that after $48 \mathrm{~h}$ treating by CPT together with the specific PI3K inhibitor-LY294002, the expression levels of cyclin A, cyclin B, cyclin D and CDK2 were further decreased in a dose-dependent manner. It significantly indicated that CPT-induced cell cycle arrest is associated with the inhibition of PI3K/AKT activity in SKBR-3 cells. IF assay also showed that a CPT induced downregulation of PI3K expression. Besides, the results by G-1 and G-15 further demonstrated that such effect was mediated by GPER. 
In conclusion, the most interesting aspect that can be extrapolated from our results is that CPT is a molecule which could be successful employed as therapeutics adjuvant against nER negative breast cancer cells. Such effect of CPT was realized by its GPER mediated regulatory function on PI3K/AKT signaling pathways.

\section{Declarations}

GPER, G protein-coupled estrogen receptor; CDK, cyclin-dependent kinase; DAPI, diamidino-2-phenylindole; FCM, flow cytometer; FITC, fluorescein isothiocyanate; PI, propidium iodide; PI3K, phosphatidylinositide 3-kinase

Acknowledgments

The authors would like to thank School of Life Sciences, Beijing University of Chinese Medicine, China for providing laboratory facilities.

Funding

The present study was supported by National Natural Science Foundation of China (No. 81673764), the 111 project from the ministry of education and bureau of foreign experts affairs of China (No. B070070and the Graduate program from Beijing University of Chinese medicine (No. 2018-JYB-XS100)

Availability of data and materials

The datasets used and analyzed during the current study are available from the corresponding author on reasonable request.

Authors' contributions

DS and PZ designed the study. DS and LC performed the experiments. DS, HL, MC and LS analyzed the data. DS and PZ wrote the paper. All authors read and approved the final manuscript.

Ethics approval and consent to participate

Not applicable.

Consent for publication

Not applicable.

Conflict of Interests

The authors declare that they have no competing interests.

\section{References}

1. Harbeck N, Gnant M: Breast cancer. LANCET 2017, 389(10074):1134-1150. 
2. Abderrahman B, Jordan VC: Successful Targeted Therapies for Breast Cancer, The Worcester Foundation and Future Opportunities in Women's Health. ENDOCRINOLOGY 2018.

3. Tong C, Wu M, Cho W, To K: Recent Advances in the Treatment of Breast Cancer. FRONT ONCOL 2018, 8:227.

4. Chen X, Guo J, Bao J, Lu J, Wang Y: The anticancer properties of Salvia miltiorrhiza Bunge (Danshen): a systematic review. MED RES REV 2014, 34(4):768-794.

5. Gong Y, Li Y, Abdolmaleky HM, Li L, Zhou JR: Tanshinones inhibit the growth of breast cancer cells through epigenetic modification of Aurora A expression and function. PLOS ONE 2012, 7(4):e33656.

6. Nicolin V, Fancellu G, Valentini R: Effect of tanshinone II on cell growth of breast cancer cell line type MCF7 and MD-MB-231. Ital J Anat Embryol 2014, 119(1):38-43.

7. Wang L, Wu J, Lu J, Ma R, Sun D, Tang J: Regulation of the cell cycle and PI3K/Akt/mTOR signaling pathway by tanshinone I in human breast cancer cell lines. MOL MED REP 2015, 11(2):931-939.

8. Tang S, Shen XY, Huang HQ, Xu SW, Yu Y, Zhou CH, Chen SR, Le K, Wang YH, Liu PQ: Cryptotanshinone suppressed inflammatory cytokines secretion in RAW264.7 macrophages through inhibition of the NFkappaB and MAPK signaling pathways. INFLAMMATION 2011, 34(2):111-118.

9. Cha JD, Lee JH, Choi KM, Choi SM, Park JH: Synergistic Effect between Cryptotanshinone and Antibiotics against Clinic Methicillin and Vancomycin-Resistant Staphylococcus aureus. Evid Based Complement Alternat Med 2014, 2014:450572.

10. Cai Y, Zhang W, Chen Z, Shi Z, He C, Chen M: Recent insights into the biological activities and drug delivery systems of tanshinones. Int J Nanomedicine 2016, 11:121-130.

11. Jiang G, Liu J, Ren B, Zhang L, Owusu L, Liu L, Zhang J, Tang Y, Li W: Anti-tumor and chemosensitization effects of Cryptotanshinone extracted from Salvia miltiorrhiza Bge. on ovarian cancer cells in vitro. J ETHNOPHARMACOL 2017, 205:33-40.

12. Chen W, Lu Y, Chen G, Huang S: Molecular evidence of cryptotanshinone for treatment and prevention of human cancer. Anticancer Agents Med Chem 2013, 13(7):979-987.

13. Yue W, Yager JD, Wang JP, Jupe ER, Santen RJ: Estrogen receptor-dependent and independent mechanisms of breast cancer carcinogenesis. STEROIDS 2013, 78(2):161-170.

14. Farzaneh S, Zarghi A: Estrogen Receptor Ligands: A Review (2013-2015). Sci Pharm 2016, 84(3):409-427.

15. Jameera BA, Jubie S, Nanjan MJ: Estrogen receptor agonists/antagonists in breast cancer therapy: A critical review. BIOORG CHEM 2017, 71:257-274.

16. Prossnitz ER, Barton M: Estrogen biology: new insights into GPER function and clinical opportunities. MOL CELL ENDOCRINOL 2014, 389(1-2):71-83.

17. Pupo M, Maggiolini M, Musti AM: GPER Mediates Non-Genomic Effects of Estrogen. Methods Mol Biol 2016, 1366:471-488.

18. Molina L, Figueroa CD, Bhoola KD, Ehrenfeld P: GPER-1/GPR30 a novel estrogen receptor sited in the cell membrane: therapeutic coupling to breast cancer. Expert Opin Ther Targets 2017, 21(8):755-766. 
19. Wei W, Chen ZJ, Zhang KS, Yang XI, Wu YM, Chen XH, Huang HB, Liu HL, Cai SH, Du J et al: The activation of $\mathrm{G}$ protein-coupled receptor 30 (GPR30) inhibits proliferation of estrogen receptor-negative breast cancer cells in vitro and in vivo. CELL DEATH DIS 2014, 5:e1428.

20. Asghar U, Witkiewicz AK, Turner NC, Knudsen ES: The history and future of targeting cyclin-dependent kinases in cancer therapy. NAT REV DRUG DISCOV 2015, 14(2):130-146.

21. Fu J, Tian C, Xing M, Wang X, Guo H, Sun L, Sun L, Jiang Z, Zhang L: KU004 induces G1 cell cycle arrest in human breast cancer SKBR-3 cells by modulating PI3K/Akt pathway. BIOMED PHARMACOTHER 2014, 68(5):625-630.

22. Ge X, Guo R, Qiao Y, Zhang Y, Lei J, Wang X, Li L, Hu D: The G protein-coupled receptor GPR30 mediates the nontranscriptional effect of estrogen on the activation of PI3K/Akt pathway in endometrial cancer cells. INT J GYNECOL CANCER 2013, 23(1):52-59.

23. Kim MS, Kim JE, Lim DY, Huang Z, Chen H, Langfald A, Lubet RA, Grubbs CJ, Dong Z, Bode AM: Naproxen induces cell-cycle arrest and apoptosis in human urinary bladder cancer cell lines and chemically induced cancers by targeting PI3K. Cancer Prev Res (Phila) 2014, 7(2):236-245.

24. Zhao PW, Lee DY, Ma ZZ, Huang LS, Sun LP, Li YD, Chen JX, Niu JZ: The antioxidant effect of carnosol in bovine aortic endothelial cells is mainly mediated via estrogen receptor alpha pathway. BIOL PHARM BULL 2012, 35(11):1947-1955.

25. Akram M, Iqbal M, Daniyal M, Khan AU: Awareness and current knowledge of breast cancer. BIOL RES 2017, 50(1):33.

26. Bhat MA, Al-Dhfyan A, Naglah AM, Khan AA, Al-Omar MA: Lead Optimization of 2-Cyclohexyl-N-[(Z)-(3methoxyphenyl/3-hydroxyphenyl) methylidene]hydrazinecarbothioamides for Targeting the HER-2 Overexpressed Breast Cancer Cell Line SKBr-3. MOLECULES 2015, 20(10):18246-18263.

27. Lappano R, Pisano A, Maggiolini M: GPER Function in Breast Cancer: An Overview. Front Endocrinol (Lausanne) 2014, 5:66.

28. Steiman J, Peralta EA, Louis S, Kamel O: Biology of the estrogen receptor, GPR30, in triple negative breast cancer. AM J SURG 2013, 206(5):698-703.

29. Weissenborn C, Ignatov T, Poehlmann A, Wege AK, Costa SD, Zenclussen AC, Ignatov A: GPER functions as a tumor suppressor in MCF-7 and SK-BR-3 breast cancer cells. J Cancer Res Clin Oncol 2014, 140(4):663671.

30. Ariazi EA, Brailoiu E, Yerrum S, Shupp HA, Slifker MJ, Cunliffe HE, Black MA, Donato AL, Arterburn JB, Oprea TI et al: The G protein-coupled receptor GPR30 inhibits proliferation of estrogen receptor-positive breast cancer cells. CANCER RES 2010, 70(3):1184-1194.

31. Chimento A, Casaburi I, Rosano C, Avena P, De Luca A, Campana C, Martire E, Santolla MF, Maggiolini M, Pezzi V et al: Oleuropein and hydroxytyrosol activate GPER/ GPR30-dependent pathways leading to apoptosis of ER-negative SKBR3 breast cancer cells. MOL NUTR FOOD RES 2014, 58(3):478-489.

32. Ariazi EA, Brailoiu E, Yerrum S, Shupp HA, Slifker MJ, Cunliffe HE, Black MA, Donato AL, Arterburn JB, Oprea TI et al: The G protein-coupled receptor GPR30 inhibits proliferation of estrogen receptor-positive breast cancer cells. CANCER RES 2010, 70(3):1184-1194. 
33. Wang C, Lv X, Jiang C, Davis JS: The putative G-protein coupled estrogen receptor agonist G-1 suppresses proliferation of ovarian and breast cancer cells in a GPER-independent manner. AM J TRANSL RES 2012, 4(4):390-402.

34. Dennis MK, Burai R, Ramesh C, Petrie WK, Alcon SN, Nayak TK, Bologa CG, Leitao A, Brailoiu E, Deliu E et al: In vivo effects of a GPR30 antagonist. NAT CHEM BIOL 2009, 5(6):421-427.

35. Chen J, Shi DY, Liu SL, Zhong L: Tanshinone IIA induces growth inhibition and apoptosis in gastric cancer in vitro and in vivo. ONCOL REP 2012, 27(2):523-528.

36. Choi YJ, Anders L: Signaling through cyclin D-dependent kinases. ONCOGENE 2014, 33(15):1890-1903.

37. Aaltonen K, Amini RM, Heikkila P, Aittomaki K, Tamminen A, Nevanlinna H, Blomqvist C: High cyclin B1 expression is associated with poor survival in breast cancer. Br J Cancer 2009, 100(7):1055-1060.

38. Manning BD, Toker A: AKT/PKB Signaling: Navigating the Network. CELL 2017, 169(3):381-405.

39. Ahmad A, Biersack B, Li Y, Kong D, Bao B, Schobert R, Padhye SB, Sarkar FH: Deregulation of PI3K/Akt/mTOR signaling pathways by isoflavones and its implication in cancer treatment. Anticancer Agents Med Chem 2013, 13(7):1014-1024.

40. Schettini F, Buono G, Trivedi MV, De Placido S, Arpino G, Giuliano M: PI3K/mTOR Inhibitors in the Treatment of Luminal Breast Cancer. Why, When and to Whom? Breast Care (Basel) 2017, 12(5):290-294.

\section{Materials And Methods}

Reagents.

Cryptotanshinone (CPT, purity $[98 \%$, Fig.1A) obtained from National Institutes for Food and Drug Control (Beijing, China) was dissolved in dimethyl sulfoxide (DMSO, Sigma, USA) in a stock concentration of 10mM at $-20{ }^{\circ} \mathrm{C}$. The methyl thiazolyl tetrazolium (MTT) was obtained from Keygen (Nanjing, China). RPMI-1640 medium and $0.05 \%$ trypsin were from Gibco (USA) and Fetal bovine serum was from Corning Cell Gro (Australia).

Lipofectamine ${ }^{\circledR} 2000$ was from Invitrogen (Grand Island, NY, USA). GPER specific agonist G-1 and antagonist G15 were from Cayman Chemical (Michigan, USA). The following antibodies were used: GPER, PI3K(p85), cyclin A, cyclin B, cyclin D, CDK2 (Abcam, USA), p-AKT (Cell signaling, Boston, MA, USA). GPER siRNA, non-target siRNA and PI3K inhibitor LY294002 were obtained from Santa Cruz Biotechnology (Texas USA). FluoresceinConjugated Goat anti-Rabbit IgG $₫ \mathrm{H}+\mathrm{L} \rrbracket$ was from ZSGB-BIO (Beijing, China) and DAPI was from Solarbio (Beijing, China).

Cell Culture.

The human breast cancer ER negative SKBR-3 cells obtained from the National Infrastructure of Cell Line Resource (Beijing, China) were maintained in RPMI-1640 medium supplemented with 10\% fetal bovine serum, $100 \mu \mathrm{g} / \mathrm{ml}$ streptomycin and $100 \mathrm{U} / \mathrm{ml}$ penicillin at $37^{\circ} \mathrm{C}$ in a $5 \% \mathrm{CO}_{2}$ atmosphere.

MTT cell viability assay. 
MTT (3-[4,5-dimethylthiazol-2-yl]-2,5-diphenyltetrazolium bromide) cell viability assay was performed as already described[24]. Briefly, cells were seeded in 96-well plates at a density of $5 \times 10^{3}$ cells per well in the growth medium. After $12 \mathrm{~h}$, the cells were treated with 1-10 $\mathrm{mmol} / \mathrm{L} \mathrm{CPT}$ or 0.2\% DMSO as a control. 24 or $48 \mathrm{~h}$ later, fifteen micro liter MTT reagent $(1 \mathrm{mg} / \mathrm{mL})$ was added into each well and the plates were incubated for an additional $4 \mathrm{~h}$ in the dark. Subsequently, the supernatants were removed and cells were lysed in 150 $\mu \mathrm{l}$ DMSO. The optical density was at a measurement wavelength of $490 \mathrm{~nm}$ and a reference wavelength of $570 \mathrm{~nm}$ using a plate reader (Multiskan GO, ThermoFisher Scientific, USA).

\section{Analysis of cell cycle distribution.}

After treatment with 5-10 $\mu \mathrm{M}$ CPT for $48 \mathrm{~h}$, SKBR-3 cells were removed using 0.05\% trypsin, washed and stained with $50 \mu \mathrm{g} / \mathrm{ml}$ PI (Sigma, USA) and $250 \mu \mathrm{g} / \mathrm{ml}$ RNase in PBS buffer for 30 min at room temperature in the dark. Subsequently, fluorescence activated cell sorting analysis was performed by FCM (BD FACSCanto II, Becton, Dickinson and Company, NJ, USA).

\section{Small interfering RNA (siRNA) transfection.}

SKBR-3 cells were seeded in 6-well cell culture plates. At 80-90\% confluent, the cells were transfected with 33nmol/L GPER siRNA or labeled non-target siRNA as control using Lipofectamine ${ }^{\circledR} 2000$ transfection reagent according to the manufacture's instruction. After $24 \mathrm{~h}$, the medium was replaced with fresh medium and the cells were cultured for an additional $24 \mathrm{~h}$ before Western blot analysis of GPER expression and MTT cell viability assay.

\section{Western blot analysis.}

Exponentially growing cells were seeded in a 6-well plate. $24 \mathrm{~h}$ after seeding, the SKBR-3 cells in the logarithmic growth phase were incubated in the absence or presence of CPT with or without specific GPER agonist G-1 and antagonist G-15 for the indicated duration. The cells were washed twice with ice-cold phosphate-buffered saline (PBS) and placed in RIPA buffer (Applygen, Beijing, China) supplemented with 1\% protein phosphatase inhibitor (All-in-one,100x, Solarbio, Beijing, China) immediately prior to use. Total cell extracts were severally collected in different centrifuge tubes and centrifuged at $12000 \mathrm{rpm}$ for $10 \mathrm{~min}$ at $4^{\circ} \mathrm{C}$. Supernatants were recovered subsequently. Then the protein concentration was quantified using bicinchoninic acid (BCA) protein assay kit (Solarbio, Beijing, China). Equal quantities of protein $(50 \mu \mathrm{g})$ from each sample were separated by $10 \%$ SDSpolyacrylamide gel electrophoresis (PAGE) and then transferred to polyvinylidene fluoride (PVDF) membranes. The membranes were blocked with 5\% skim milk dissolved in tris-buffered saline (TBS)-T (1X TBS, 0.1\%Tween 20) for $2 \mathrm{~h}$ at room temperature. Western blot analysis was performed using the following primary antibody dilutions: Rabbit anti human cyclin A $(1: 1,000)$, cyclin B $(1: 10,000)$, cyclin D $(1: 10,000)$, CDK2 $(1: 10,000)$, PI3K $(1: 1,000)$, GPER $(1: 250)$ and mouse anti $\beta$-actin $(1: 10,000)$ was diluted in $5 \%$ skim milk respectively. In addition,

rabbit anti human p-AKT was diluted for 1:2000 in 5\% BSA according to introduction. Then the membranes were incubated with the above primary antibody dilutions overnight at $4^{\circ} \mathrm{C}$ followed by the secondary antibody horseradish peroxidase-labeled goat anti-rabbit IgG or goat anti-mouse IgG (1:5000, Proteintech Group, Inc, China) incubation at $37^{\circ} \mathrm{C}$ for $1 \mathrm{~h}$. After additional washes, the membranes were $\mathrm{using}$ an enhanced chemiluminescence kit (ECL, Applygen, Beijing, China) and scanned in the multifunctional molecular imaging 
system (Azure C-Series C600, USA). At last, the bands were collected and analyzed by Image J software (version 1.48, National Institutes of Health, USA).

Immunofluorescence (IF) assay.

Cells were seeded in 8 well chamber slide system (Thermos Fisher, NY, USA) at a density of $1 \times 10^{4}$ per well. Then the cells in the logarithmic growth phase were treated with indicated concentration of CPT with or without G-1 or G-15. Following incubation for another $24 \mathrm{~h}$, the cells were fixed with $4 \%$ paraformaldehyde for 30min, permeabilized with $0.02 \%$ Triton-X-100 for $20 \mathrm{~min}$ and blocked with $5 \%$ goat serum for 30 min. Then the cells were incubated with specific primary antibodies at $4^{\circ} \mathrm{C}$ overnight followed by fluorescein isothiocyanate (FITC) labeled secondary antibody for $1 \mathrm{~h}$ away from light at room temperature. After washed with PBS, DAPI was used for nucleus staining. Images were visualized using an inverted fluorescence microscope (IX71, Olympus, Japan) and the mean fluorescence intensity was analyzed by Image J.

Statistical analysis.

Data are expressed as mean \pm S.D. of three independent experiments. Data were analyzed by one-way ANOVA for multiple comparison involved. The statistical analyses were performed using SPSS 20.0 (SPSS, Inc, Chicago, IL, USA) for Windows. The significant difference was confirmed with $P \square 0.05$.

\section{Figures}


A.

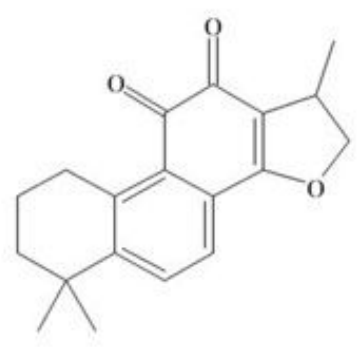

B.

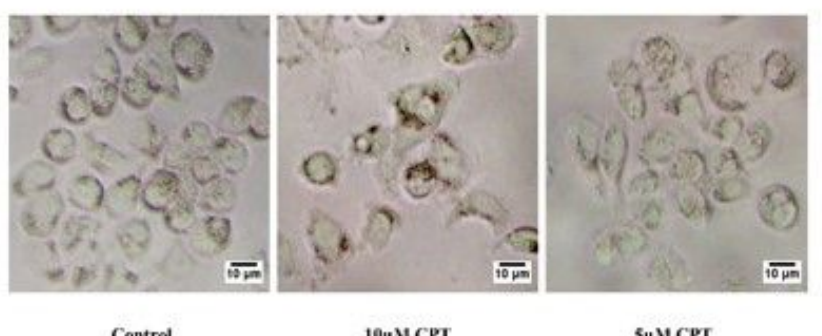

$s_{\mu} \mathrm{MCPT}$

c.

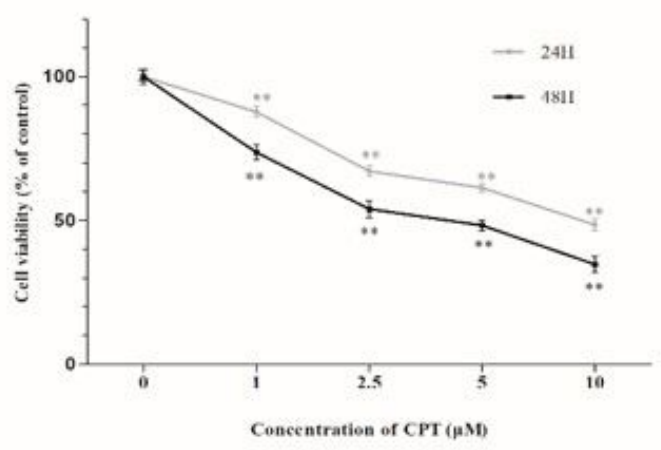

Figure 1

Inhibitory effect of CPT on SKBR-3 cell viability. (A) Chemical structure of Cryptotanshinone (CPT, molecular weight $=296.35 \mathrm{~g} / \mathrm{mol}$ ). (B) SKBR-3 cell morphology under inverted microscope (magnification, $\times 200$ ) after CPT treating for $24 \mathrm{~h}$ with $0.2 \%$ DMSO as control. Scale bar represents $10 \mu \mathrm{m}$. (C) SKBR-3 cells were treated with indicated concentrations of CPT for $24 \mathrm{~h}$ or $48 \mathrm{~h}$ and cell viability was tested using MTT

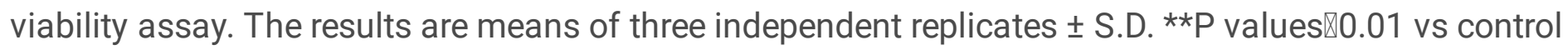
group were considered as statistically significant. 
A.

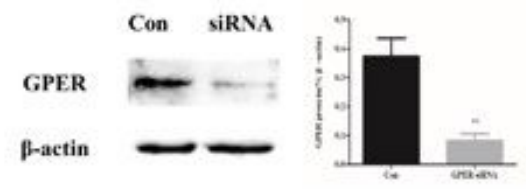

B.

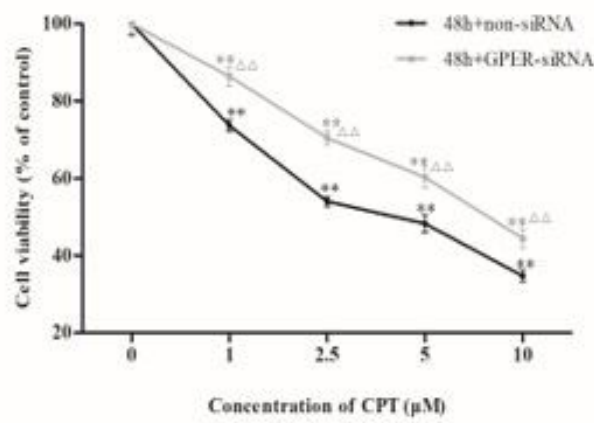

c.

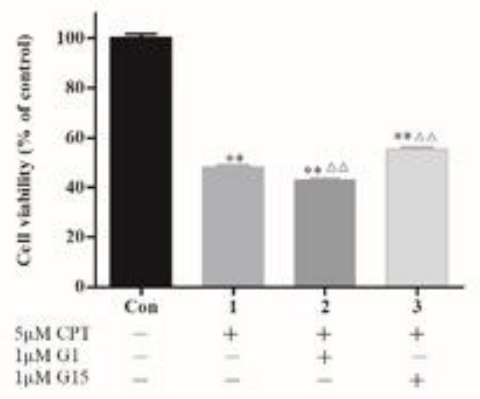

\section{Figure 2}

CPT inhibits SKBR-3 cell viability via GPER mediated pathway. (A) GPER protein expression level in GPER siRNA transfected SKBR-3 cells were tested by Western blot with non-siRNA transfection as control. **PQ 0.01 vs control group. (B) Viability of GPER knocked down SKBR-3 cells was tested by MTT viability

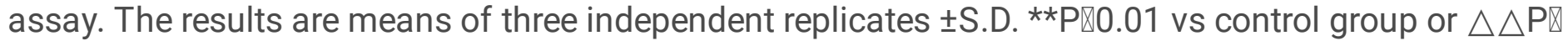
0.01 vs non-siRNA group were considered as statistically significant. (C) Viability of SKBR-3 cells treated by CPT with $\mathrm{G} 1$ or $\mathrm{G} 15$ was also tested by MTT viability assay. The results are means of three independent replicates \pm S.D. ${ }^{*} \mathrm{P} \otimes 0.01$ vs control group or $\triangle \triangle \mathrm{P} \otimes 0.01$ vs $5 \mu \mathrm{M} C P T$ treated group were considered as statistically significant.
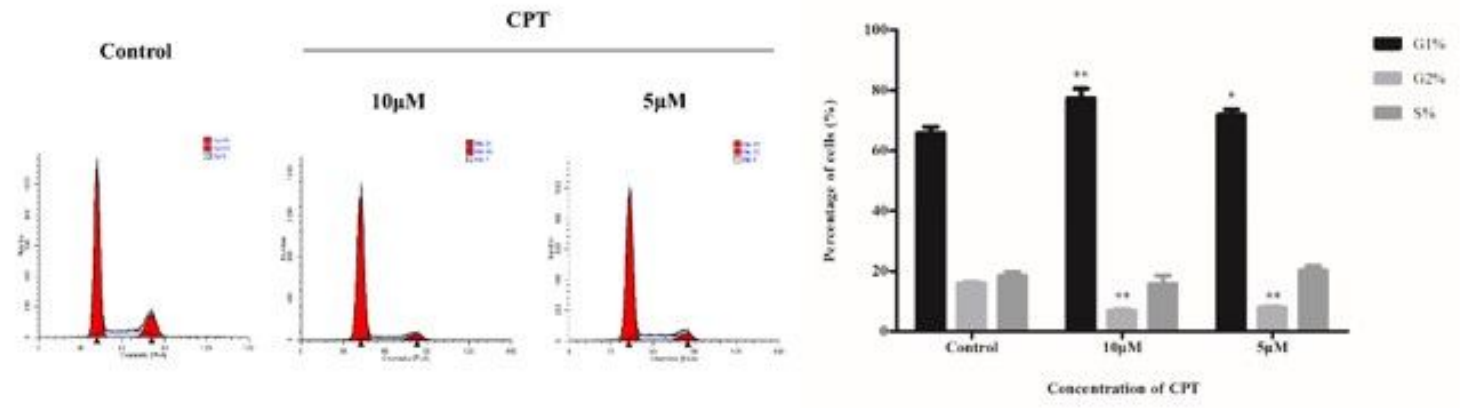

\section{Figure 3}

Effect of CPT treating for $48 \mathrm{~h}$ on cell cycle distribution in SKBR-3 cells. The results are means of three

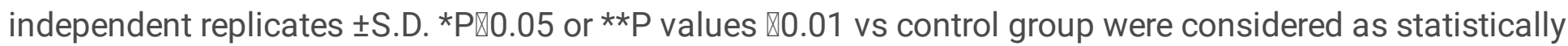
significant. 


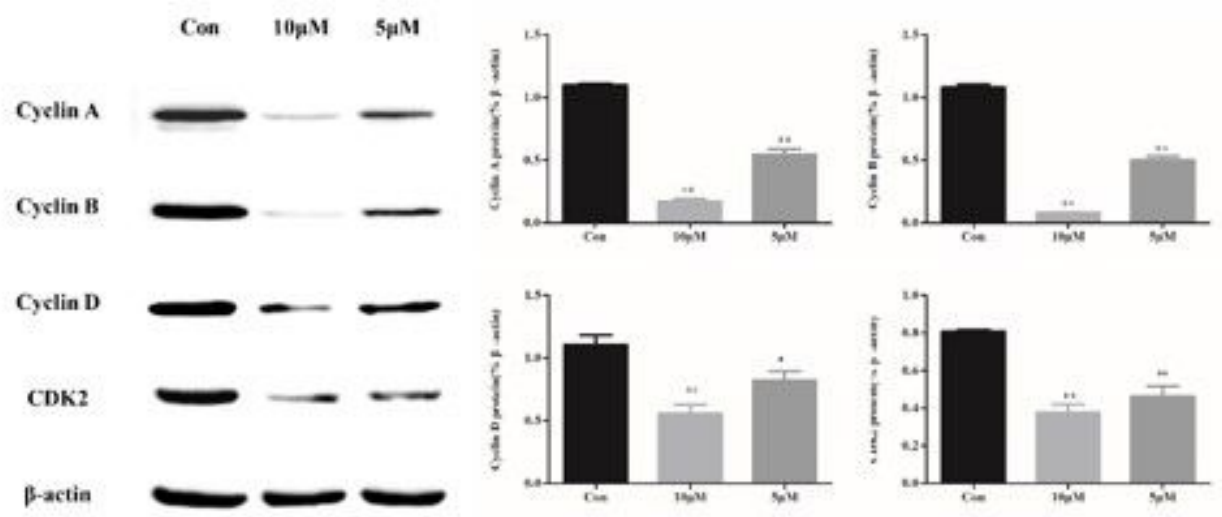

Figure 4

The expression level of cell cycle-associated proteins in SKBR-3 following treatment with CPT for $48 \mathrm{~h}$. The results are means of three independent replicates \pm S.D. $* P \otimes 0.05$ or $* \star P$ values $\otimes 0.01$ vs control group were considered as statistically significant.

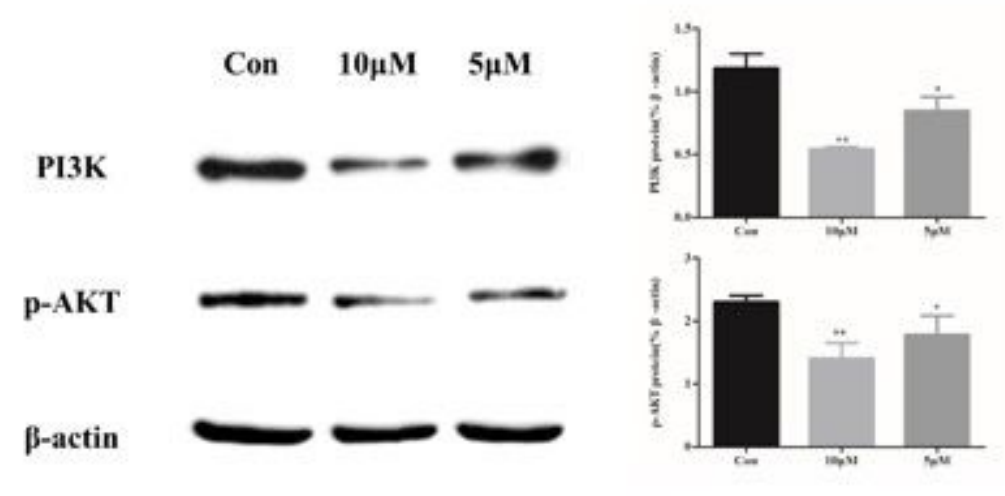

Figure 5

The expression level of PI3K and p-AKT in SKBR-3 cells treated by CPT for $48 \mathrm{~h}$. The results are means of three independent replicates \pm S.D. ${ }^{\star} \mathrm{P} \otimes 0.05$ vs control group were considered as statistically significant. 

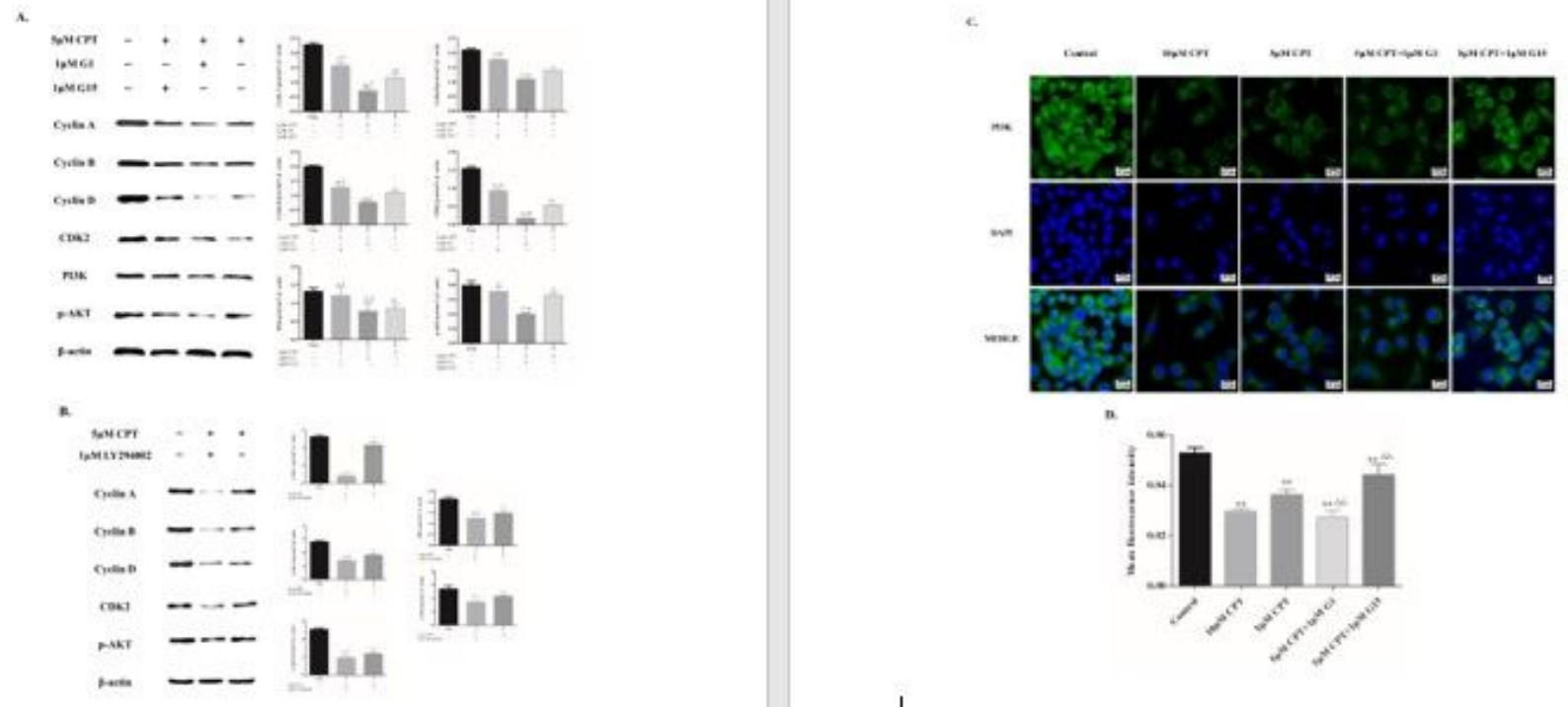

Figure 6

(A) The expression level of cyclin A, cyclin B, cyclin D, CDK2, PI3K and p-AKT following G-1, G-15 treating together with $5 \mu \mathrm{M}$ CPT. (B) The expression level of cyclin A, cyclin B, cyclin D, CDK2 and p-AKT following LY294002 treating together with $5 \mu$ M CPT. (C) Effect of CPT treatment for $24 \mathrm{~h}$ on PI3K expression by using immunofluorescence assay. Nuclei were counterstained with DAPI (blue). (magnification, $\times 200$, Scale bar represents $10 \mu \mathrm{m}$ ). (D) The mean fluorescence intensity of PI3K by IF assay was analyzed using Image $\mathrm{J}$ software. The results are means of three independent replicates \pm S.D. ${ }^{*} \mathrm{P} \otimes 0.01$ or $* \mathrm{P} \otimes 0.05$ vs control group, $\triangle \triangle \mathrm{P} \otimes 0.01$ vs $5 \mu \mathrm{M} C P T$ treating group were considered as statistically significant. 